TITLE:

\title{
Genetic consequences of rapid population decline and restoration of the critically endangered herb Polemonium kiushianum
}

\section{$\operatorname{AUTHOR(S):~}$}

Yokogawa, Masashi; Kaneko, Shingo; Takahashi, Yoshitaka; Isagi, Yuji

\section{CITATION:}

Yokogawa, Masashi ...[et al]. Genetic consequences of rapid population decline and restoration of the critically endangered herb Polemonium kiushianum. Biological Conservation 2013, 157: 401-408

ISSUE DATE:

2013-01

URL:

http://hdl.handle.net/2433/169032

\section{RIGHT:}

(c) 2012 Elsevier Ltd.; この論文は出版社版でありません。引用の際には 出版社版をご確認ご利用ください。; This is not the published version. Please cite only the published version. 
1 Genetic consequences of rapid population decline and restoration of the critically endangered

3 Masashi Yokogawa $^{1 *}$, Shingo Kaneko ${ }^{1}$, Yoshitaka Takahasi ${ }^{2}$, Yuji Isagi ${ }^{1}$

4

(1) Graduate School of Agriculture, Kyoto University, Oiwake-cho, Kitashirakawa, Sakyo-ku, Kyoto 606-8502, Japan.

(2) National Agricultural Research Center for Western Region, Ohda, Shimane 694-0013, Japan.

*Corresponding author : Yuji Isagi

Graduate School of Agriculture, Kyoto University, Oiwake-cho, Kitashirakawa, Sakyo-ku, Kyoto 606-8502, Japan.

Tel: +81 75753 6129, Fax: 8175753 6129, E-mail: yokogawa@kais.kyoto-u.ac.jp

\section{Abstract}

Many critically endangered species require not only in situ but also ex situ conservation to reduce extinction risk. In this study, all five known wild populations and two artificially managed ex situ populations outside the species' native range of Polemonium kiushianum, a critically endangered herb species in Japan, were studied, using 10 polymorphic microsatellite markers to assess the genetic consequences of habitat degradation on the wild populations and the establishment of ex situ populations. Levels of genetic diversity among the wild populations were similar to each other, whereas genetic diversity in the ex situ populations was considerably lower than that of the wild populations. A significant level of genetic differentiation was associated with a recent bottleneck and genetic drift in the wild populations. The STRUCTURE 
analysis revealed that the genetic composition of the two ex situ populations differed from that

of the five wild populations. The low genetic diversity observed in the ex situ populations and different genetic composition between wild and ex situ populations may be due to genetic drift with few founders for the ex situ populations and the management strategy used for the ex situ populations. Seed transfer simulations using simulated genotypes generated on computer programs indicated that 1,000 or more seeds were needed to enhance genetic diversity and recover the genetic composition of the present ex situ populations. These simulations used to predict the genetic consequences of immigration represent a powerful tool for conservation management of critically endangered species based on genetic data.

Keyword: ex situ conservation, genetic drift, genetic structure, microsatellite, Polemonium kiushianum, seed transfer simulation

\section{Introduction}

Many plant species throughout the world are threatened with extinction due to habitat destruction and fragmentation as a result of human activities. Populations of endangered species

41 in their natural habitat are vulnerable to loss of genetic diversity due to a decline and/or

42 fluctuation in population sizes, resulting in reduced gene flow among remnant populations,

43 inbreeding, and genetic drift (Lowe et al., 2005; Frankham et al., 2010). Empirical and experimental studies also indicate that demographic decline and population bottlenecks cause or contribute to a loss of genetic diversity (Cornuet and Luikart, 1996; Young et al., 1996). The loss of genetic diversity associated with demographic decline can affect the viability of the

47 population in short term (Keller and Waller 2002) and limitations in the species' ability to respond to the changing environment in long term (Young et al 1996). 
Although preservation of the natural habitat is critical for the conservation of

ecological interactions, ex situ measures may be appropriate for safeguarding individual species against extinction in the wild (Russello and Amato, 2007). The primary purpose of ex situ conservation is to maintain wild species outside their natural habitat so that species recovery and reintroduction can be attempted in the case of severe decline or extinction of wild populations (Husband and Campbell, 2004). However, despite the significance of ex situ conservation in endangered species management, these populations are exposed to forces similar to those encountered by in situ populations, which may decrease genetic diversity. For example, ex situ populations usually have a restricted founder source and small population size and are susceptible to inbreeding and inbreeding depression (Frankel and Soulé, 1981; Williams et al., 2002). Low genetic diversity in ex situ populations is likely to increase extinction risk to an equal extent as seen in wild populations. is achieved by the immigration of additional breeding stock from wild populations. Moreover, information regarding the number of these stocks needed to enhance the genetic diversity of ex situ populations is an important consideration. However, immigration from wild to ex situ populations increases the short-term risk of extinction of wild populations by removing

66 individuals (Menges et al., 2004). Thus, prior prediction of the effects of immigration of

67 additional breeding stocks by simulation approaches prior to their removal from wild populations will provide useful information regarding the quantities of additional breeding stocks that are required for recovery of genetic diversity in ex situ populations. endemic to the semi-natural grasslands of the Aso region of Kyushu, Japan (Environment 
73 loss as a result of conifer plantation establishment and the abandonment of traditional grassland management (Sei, 2006). Only a few hundred individuals and five populations of this species remain, and most subpopulations have become locally extinct in the last three decades. As a result, $P$. kiushianum has been categorized as 'critically endangered' (CR) in the Japanese Red Data Book, and is now protected by the 'Law for the Conservation of Endangered Species of Wild Fauna and Flora' in Japan. In response to this situation, ex situ populations outside the species' native range were founded to provide for the degradation of the wild populations. They show promise as a temporary pool of genetic diversity that can be used to bolster wild populations. Polemonium kiushianum provides an excellent model for comparing the impact on genetic diversity that population decline of wild populations and the recent establishment of ex situ populations has made, and allows for an examination of the potential use of simulations to predict the impact of seed transfer for enhancing the genetic diversity of ex situ populations. In the present study, the genetic status of all remaining wild populations of $P$. kiushianum and its ex situ populations were assessed using polymorphic microsatellite markers. The study aimed to evaluate the following: (1) the genetic diversity, genetic structure, and recent demographic history in wild populations; (2) the genetic consequences of the foundation of ex situ populations; and (3) the possibility of recovering genetic diversity in ex situ populations by seed transfer from the remaining wild populations using simulated genotypes generated on a computer program.

\section{Material and methods}

\subsection{Study site and species}

The Aso region is located in central Kyushu, south-western Japan (Fig. 1), and consists 
97 of central volcanic mountains (up to 1,592 m above sea level [a.s.1.]) and surrounding somma

98 (800-1,100 m a.s.l.). The mean annual temperature and annual precipitation between 1992 and

992011 were $13.4^{\circ} \mathrm{C}$ and 2,386 mm, respectively (Takamori Meteorogical Observation Station, at

$100555 \mathrm{~m}$ a.s.l.). In the Aso region, vast semi-natural grasslands have been maintained by

101 human-related influences such as hand mowing, artificial burning, and livestock grazing

102 (Takahashi, 2009). Phytolith and microscopic charcoal analyses have shown that grasslands in

103 this region have been established for over 10,000 years, and formation of these grasslands has

104 been attributed to burning as a consequence of anthropogenic activities (Miyabuchi et al., 2012).

105 Since vast grassland vegetation has been maintained, the Aso region provides a habitat for rare

106 grassland herbs such as Echinops setifer, Viola orientalis, and Campanula glomerata var.

107 dahurica (Hotta, 1974), and has high grassland plant species diversity. However, due to

108 abandonment of the traditional management of grasslands and land-use change from grasslands

109 to conifer plantations or pastures, the area of the semi-natural grasslands has decreased

110 dramatically in recent years and, the populations of many grassland plant species have

111 undergone a severe decline (Takahashi, 2009).

112 Polemonium kiushianum is an endangered plant of eastern somma of the Aso region.

113 This species is a diploid $(2 \mathrm{n}=18$, Inaba et al., 2010) perennial herb that occurs in sunny

114 meadows maintained by mowing at altitudes of approximately $700-900 \mathrm{~m}$. This species is not a

115 clonal plant and the individual longevity might be 3-4 years, with a maximum of up to 10 years

116 (Yokogawa et al., unpublished results). Pollinators of this species are bumblebees or small

117 solitary bees (Yokogawa et al., unpublished results) and seeds are dispersed by gravity. Whereas

11846 wild populations were identified from 1988 to 1993 (Sei, 2006), only seven wild populations

119 were found in 2004 (Matoba et al., 2011). This number had declined to only five wild

120 populations by 2009 (Fig. 1; Table 1). The habitats of three of the remaining five wild 
121 populations have been degraded by artificial conifer plantations (mainly Japanese cedar, Cryptomeria japonica (L.f.) D. Don), and thus the population size is very small (Table 1; population W3, W4, and W5). In contrast, the other two populations that remain in mowed grassland have relatively large population sizes (Table 1; population W1 and W2). However, the sizes of these populations were very small 10 years ago due to the abandonment of mowing.

126 After the population decline, conservation activities such as the restart of mowing were

127 conducted, and the population sizes subsequently recovered. The two ex situ populations were 128 established outside the species' native range, and from seeds collected from the W2 and W3

129 populations in 1999-2001 (Table 1; population E6 and E7). The seed sources of each ex situ

130 population, whether from one seed source population (W2 or W3) or both seed source populations (W2 and W3), could not be clearly distinguished. Populations E6 and E7 are about $5 \mathrm{~km}$ and $25 \mathrm{~km}$ away from the edge of the species' native range, respectively. The growing environment of P. kiushianum in E6 is the deciduous forest floor with mowing in autumn and that of E7 is sunny grasslands with mowing in autumn.

\subsection{Sampling and microsatellite analysis}

138 from the five wild populations (Fig. 1) and the two ex situ populations. In large populations

139 without sampling restriction, we randomly selected over 20 individuals spaced at least $2 \mathrm{~m}$ apart,

140 or all individuals if less than 20 were present (in population W3). The population size and

141 sample size of each population are indicated in Table 1 and Table 2, respectively. Genomic DNA

142 was extracted using a modified CTAB method (Milligan, 1992).

143 The genotypes of each individual were characterized at 10 microsatellite loci. Seven

144 out of the 10 loci were developed by Yokogawa et al. (2009): Pkiu006, Pkiu059, Pkiu129, 
145 Pkiu135, Pkiu208, Pkiu212, and Pkiu227. We designed three additional microsatellite primer

146 pairs, Pkiu593, Pkiu627, and Pkiu965 (Table A.1), using the same protocol as Yokogawa et al.

147 (2009). The PCR amplifications were performed following the standard protocol of the Qiagen

148 Multiplex PCR kit (Qiagen), in a final volume of $6 \mu \mathrm{L}$, which contained $5 \mathrm{ng}$ of extracted DNA,

$1493 \mu \mathrm{L}$ of $2 \times$ Multiplex PCR Master Mix, and $0.2 \mathrm{mmol} / \mathrm{L}$ of each multiplexed primer. The PCR

150 amplifications were carried out with a GeneAmp PCR System 2700 thermal cycler (Applied

151 Biosystems), using the following conditions: initial denaturation at $95^{\circ} \mathrm{C}$ for $15 \mathrm{~min}$, followed

152 by 28 cycles of denaturation at $94^{\circ} \mathrm{C}$ for $30 \mathrm{~s}$, annealing of the designed specific primers at the

153 designated temperatures for $1 \min 30 \mathrm{~s}$, extension at $72^{\circ} \mathrm{C}$ for $1 \mathrm{~min}$, and final extension at $60^{\circ} \mathrm{C}$

154 for $30 \mathrm{~min}$. The sizes of the PCR products were measured using an ABI PRISM 3100 Genetic

155 Analyser and Genotyper software (Applied Biosystems).

156

\subsection{Statistical analysis of genetic diversity and structure}

For each population, the genetic diversity was evaluated in terms of Nei's unbiased expected heterozygosity $\left(H_{\mathrm{E}} ;\right.$ Nei, 1987$)$ and observed heterozygosity $\left(H_{\mathrm{O}}\right)$, the average number

160 of alleles per locus $(A)$, allelic richness (AR; El Mousadik and Petit, 1996), the summed number

161 of rare alleles with frequencies less than $5 \%$ among the total population $(R A)$, the summed

162 number of private alleles that are only present in a single population $(\mathrm{Pr})$, and the fixation index

163 ( $\left.F_{\text {IS }}\right)$. With the exception of the numbers of rare and private alleles, all of these parameters were

164 calculated using FSTAT ver. 2.9.3 software (Goudet, 2001). Deviation from Hardy-Weinberg

165 equilibrium was determined using FSTAT.

166 Recent bottlenecks in the populations were evaluated by BOTTLENECK ver. 1.2.02

167 (Piry et al., 1999). We simulated equilibrium conditions (10,000 replications) assuming the 168 infinite allele mutation model (IAM) and the two-phase model (TPM, mutations with 95\% 
169 single-step mutations and 5\% multistep mutations, with a variance among multiple steps of

170 approximately 12). We used the Wilcoxon signed rank test to determine a significant excess of

171 heterozygosity. Population W3 was excluded from this analysis due to its small sample size.

172 To estimate the genetic differentiation among populations, $F_{\mathrm{ST}}$ values (Weir and

173 Cockerham, 1984) were calculated. The significance of $F_{\text {ST }}$ values was tested by comparison to

174 the $95 \%$ confidence intervals derived from 1,000 bootstrap permutations. Pairwise $F_{\text {ST }}$ values

175 were calculated by randomizing multilocus genotypes between two populations with Bonferroni

176 corrections. We also calculated standardized values of $G_{\text {ST }}^{\prime}$ (Hedrick, 2005) using averaged

177 values of heterozygosity within populations at Hardy-Weinberg equilibrium $\left(H_{\mathrm{S}}\right)$, the expected

178 heterozygosity of all populations pooled $\left(H_{\mathrm{T}}\right)$, and genetic differentiation among populations

$179\left(G_{\mathrm{ST}}\right)$.

180 We evaluated genetic relationships among populations using Bayesian clustering

181 STRUCTURE ver. 2.3 (Prichard et al., 2000), which assigns individuals into $K$ clusters.

182 Population structure was simulated with values of $K=1-10$ under an admixture model, the

183 correlated allele frequencies model (Falush et al., 2003), and the LOCPRIOR model (Hubisz et

184 al., 2009). All runs involved 1,000,000 Markov chain Monte Carlo generations, after a burn-in

185 period of 100,000 iterations. Twenty runs were performed for each value of $K$. The number of

186 clusters was determined by comparing mean values and variability of log likelihoods in each

187 run. To select the optimal value of $K$, we also used the $\Delta K$ method (Evanno et al., 2005). The $F$

188 value, the amount of genetic drift between each cluster and a common ancestral population, and

189 the expected heterozygosity were calculated for each cluster.

190

191 2.4. The Optimization method and Simulation analysis of seed transfer from wild to ex situ

192 populations 
First, to evaluate the necessity of each wild population as seed sources to restore

194 genetic diversity in ex situ populations, we carried out optimization method with simulated annealing algorithm (Possingham et al. 2000). Although the presence-absence matrix of species was used for optimization analysis in the original paper, an allele matrix by population was used

197 as being directly analogous to species in the present study. We defined alleles as our

198 conservation goal and the optimization problem was to find the smallest number of wild

199 populations that better complement the allele composition already preserved in ex situ

200 populations (see Diniz-Filho et al. 2012). We used the selection frequency at which each

201 population appeared in 100 analyzed solutions as an indicator of the importance of the seed

202 source. We also assumed that the ex situ populations were already protected. These analyses were conducted using the Marxan software (Ball et al. 2009). populations, simulation analyses of seed translocation were conducted using the current data of both wild and ex situ populations. We generated seed genotypes based on the actual genotypes of wild populations using HYBLIDLAB (Nielsen et al., 2006) and simulated the possible changes in genetic diversity and genetic composition of the ex situ populations after the addition

209 of these seed genotypes. This analysis was undertaken using a 2-stage process. Firstly, 20 seed

210 genotypes per seed parent were generated assuming random mating using HYBRIDLAB. Each

211 seed genotype was generated from genotype data of the seed parent source population. This

212 process was undertaken for 10 independent runs, where the number of wild individuals from

213 which seeds were artificially collected ranged from 10 (200 generated genotypes) to 100 (2000

214 generated genotypes), with the sample size for each run differing by increments of 10 . Of the

215 total number of seeds generated for each run, only $20 \%$ of seeds were retained for analysis (with

216 the remaining $80 \%$ randomly deleted), a strategy simulating the $80 \%$ mortality rate of $P$. 
217 kiushianum observed by Otaki (2000). The second step involved crossing of the individuals in

218 the ex situ population with the generated seed genotypes using HYBRIDLAB. For each of the

21910 runs, $30 \mathrm{~F}_{1}$ seeds were generated and used for the analysis of genetic diversity. The genetic

220 diversity of each run was evaluated in terms of expected heterozygosity and allelic richness.

221 Simulated genotypes were analyzed for genetic structure using STRUCTURE ver. 2.3 with

222 genotypes of the wild and ex situ populations. The same procedures described above for the

223 STRUCTURE analysis were performed with $K=2$.

\section{Results}

\subsection{Genetic diversity and population bottlenecks}

Levels of genetic diversity among wild populations were similar to each other, and 182 individuals of Polemoniun kiushianum. The number of alleles per locus $(A)$ ranged from 2

232 to 13 , with an average of 5.7. The allelic richness within each population $(A R)$ ranged from 2.45

233 to 2.94 , with an average of 2.70 . The average observed heterozygosity $\left(H_{\mathrm{O}}\right)$ and expected

234 heterozygosity $\left(H_{\mathrm{E}}\right)$ within each population ranged from 0.34 to 0.47 , with an average of 0.39 ,

235 and from 0.37 to 0.47 , with an average of 0.40 , respectively (Table 2$)$. The fixation index $\left(F_{\text {IS }}\right)$

236 value did not deviate significantly from zero in any population. Twenty-eight alleles (49\%) were

237 rare alleles $(R A)$ with frequencies of less than 5\%, and 16 alleles $(28 \%)$ were private alleles $(\operatorname{Pr})$

238 that were only present in a single population. Many rare or private alleles were found in wild

239 populations, whereas only one rare and one private allele were found in the ex situ populations

240 (Table 2). BOTTLENECK (tested by Wilcoxon's signed rank test) analysis indicated recent 
241 population bottlenecks in all analysed wild populations. Under the IAM, a significant excess of

242 heterozygosity was detected in all analysed wild populations, which was not detected in the two

243 ex situ populations (Table 2). There was no excess of heterozygosity in any of the six

244 populations under the TPM (Table 2).

245

3.2 Genetic differentiation and structure of wild and ex situ populations

Significant genetic differentiation was observed among the populations. The $F_{\mathrm{ST}}$ value was 0.100 with $95 \%$ confidence intervals of 0.073 to 0.124 across all seven populations, and 0.092 with $95 \%$ confidence intervals of 0.63 to 0.126 across the five wild populations, respectively. Pairwise $F_{\mathrm{ST}}$ estimates ranged from 0.03 to 0.21 (Table A.2) and all of these were significantly larger than 0 despite the small geographic distance between the wild populations. populations. distinct genetic clusters (Fig. 2). The $\Delta K$ value representing the hierarchical approach for STRUCTURE analysis was clearly the highest at $K=3$ (Fig. 2b). Thus, $K=3$ was the uppermost hierarchical level of genetic structure. Meanwhile, although the variance of log

258 likelihood among runs was high, and the results of membership analyses were unstable and

259 multimodal among runs at $\mathrm{K} \geq 5$ (Fig. 2a), the variance of log likelihood among runs was low 260 and no multimodalities were detected at $K=4$. Therefore, $K=4$ also yielded meaningful results.

261 Consequently, the results obtained with $K=3$ and $K=4$ are shown herein (Fig. 2c). When $K=3$,

262 individuals were clearly divided into 3 clusters. Wild populations W1, W2, and W3, wild

263 populations W4 and W5, and ex situ populations E6 and E7 were assigned to cluster I, cluster II, 264 and cluster III, respectively (Fig. 2). The $F$ values of clusters I and II were lower than that of 
cluster III, and the expected heterozygosity of cluster I, II, and III was $0.46,0.43$, and 0.38 ,

respectively (Fig. 2). These results suggested that ex situ populations have undergone larger moderate, and that of cluster II-b and III was high. The expected heterozygosity of cluster I (0.45) was the highest, cluster II-b (0.42) was moderate, and that of cluster II-a (0.39) and III

271 (0.38) was the lowest.

3.3 Simulation of genetic diversity and composition of seed transfers to the ex situ population as seed source for ex situ populations to preserve all alleles in ex situ populations. Seed transfer simulations indicated that random seed transfer from wild to ex situ populations required a substantially large number of seeds in order to recover the genetic diversity and genetic composition of the ex situ population. When the number of seeds transferred from wild to ex

281 situ populations was between 600 and 1000, the allelic richness and expected heterozygosity of

282 the simulated ex situ populations reached a plateau (Fig. 3), although the number of individuals

283 in each of the two ex situ populations was approximately 100 . The assignment probability of wild clusters of simulated ex situ populations peaked when the number of seeds transferred from wild to ex situ populations was 1600 (Fig. 3). To achieve ex situ population genetic compositions that closely approximated those of the wild populations, an abundance of seeds from the wild populations was needed. 


\section{Discussion}

\subsection{Genetic characteristics and demographic history of wild populations}

Although population sizes of the large wild populations (W1 and W2) of Polemonium

292 kiushianum were more than 10 times larger than those of the small wild populations (W3, W4,

293 and W5), the levels of allelic diversity and heterozygosity among the wild populations were

294 similar to each other. In general, allelic diversity and heterozygosity are positively correlated

295 with population size (e.g. Leimu et al., 2006). However, in the presence of a population

296 undergoing size fluctuations, genetic diversity is most strongly influenced by the generation of a

297 minimum population size (Frankham et al., 2010). The relatively low genetic diversity in large wild populations W1 and W2 may be explained by fluctuations in the sizes of these populations. Small $\mathrm{W} 1$ and $\mathrm{W} 2$ population sizes were observed 10 years prior to this study due to the abandonment of mowing and short-term population bottleneck effects were also indicated by the BOTTLENECK analysis under IAM in the present study. Therefore, the genetic diversity of the large wild populations may reflect their small size observed 10 years prior to this study. On the other hand, population $\mathrm{W} 3$ has high genetic diversity with 2 private alleles compared to the

304 other populations (Table 2) despite having the smallest population size (Table 1). This

305 population had a large population size 20 years ago (Otaki 2000); this suggests that population

306 W3 is likely to harbor past genetic diversity.

307 Despite the small geographic range of P. kiushianum, there was significant genetic

308 differentiation among the wild populations. The wild populations of this species experienced

309 genetic drift and population bottleneck. Given the short longevity of this species (3-4 years and

310 up to 10 years; Yokogawa et al. unpublished results) and the drastic decrease in the semi-natural

311 grassland, including the habitat of this species, over the past century in the study area (Shoji,

312 2006), this genetic differentiation and drift are likely to have occurred in association with 
313 habitat fragmentation. Other studies on endangered plants in fragmented grassland on similar

314 geographic scales (10-20 km) suggest that genetic drift and bottlenecks can lead to population

315 genetic differences after fragmentation of grassland areas (Honnay et al., 2006; Jacquemyn et al.,

316 2010). Population differentiation and the effects of genetic drift in P. kiushianum may indicate

317 that gene flow has been disrupted by population fragmentation. Given that pollinators of this

318 species are bumblebees and small solitary bees (Yokogawa et al. unpublished data) and their

319 maximum foraging distance are several hundred meter and up to $1.5 \mathrm{~km}$ (Knight et al., 2003;

320 Zurbuchen et al., 2010), pollinator-mediated gene flow between remnant populations (minimum

321 pairwise geographic distance is $1.4 \mathrm{~km}$; Table A2) is unlikely to occur. Thus, it is important to restore local extinct populations as stepping-stones to increase gene flow between the remnant wild populations.

\subsection{Genetic diversity and composition of ex situ populations}

The genetic diversity of the ex situ populations of $P$. kiushianum was generally lower than that of the wild populations, particularly for rare and private alleles. These results indicate that the genetic diversity in ex situ populations may not be sufficient to maintain the genetic diversity of the species in the case of extinction of wild populations. In plant population genetic

330 studies comparing genetic diversity between ex situ populations and wild populations, similar

331 observed levels of genetic diversity in wild and ex situ populations have been found in some of

332 the populations that were studied, for example the short-lived herb Cynoglossum officinale

333 (Enßlin et al., 2011) and the endangered Chinese tree Vatica guangxiensis (Li et al., 2002). The

334 high genetic diversity observed in ex situ populations could be due to mating among plants from

335 several populations of different origin (Enßlin et al., 2011). In contrast, genetic diversity in ex

336 situ populations was lower than that of natural populations of transplanted eelgrass Zostera 
337 marina (Williams and Davis, 1996), fruit tree Inga edulis (Hollingsworth et al., 2005), and

338 evergreen oak Cyclobalanopsis myrsinaefolia (Liu et al., 2008). The low genetic diversity in ex

339 situ or planted populations could be due to founder effects associated with the establishment of

340 these populations. The ex situ populations of $P$. kiushianum would also have lost genetic

341 diversity during establishment.

342 The results of the STRUCTURE analyses indicated not only the reduction of genetic

343 diversity but also changes in genetic composition in the ex situ populations compared with those

344 of the wild populations. These results may have been caused by founder effects that occurred

345 when the ex situ populations were established and/or genetic drift after establishment of the ex

346 situ populations associated with small population size and management strategies. Genetic

347 differences between wild and artificial populations caused by insufficient sampling of founders

348 for artificial populations have been reported in other plant species (Li et al., 2005). Many

349 individuals in restored populations of $P$. kiushianum have been regenerated every year to obtain

350 nursery stock for markets, and alternation of generations in ex situ populations would be more

351 rapid than that in wild populations. These management strategies could drive genetic drift after

352 the establishment of ex situ populations. Population bottlenecks in ex situ populations were not

353 detected by BOTTLENECK analysis, as the ex situ populations achieved near mutation-drift

354 equilibrium (see Priy, 1999) as a consequence of management for faster alternation of

355 generations, while $F$ values (as indicators of genetic drift in the STRUCTURE analysis) were

356 high in the ex situ populations. The $F_{\mathrm{ST}}$ values for both wild and ex situ populations were higher

357 than the $F_{\text {ST }}$ values for wild populations. Moreover, although the ex situ populations E6 and E7

358 were established using seeds collected from populations W2 and W3, these populations were

359 not clustered together in the STRUCTURE analysis (Fig 2). These results also suggest that two

360 ex situ populations experienced severe genetic drift, and that the genetic composition of these 
361 populations differed substantially compared to the wild populations. The low genetic diversity and different genetic composition observed in ex situ populations may indicate genetic deterioration in ex situ populations through the process of establishment and management of

364 these populations.

\subsection{Restoration of genetic diversity in ex situ populations and effectiveness of seed transfer} simulation

The genetic deterioration in ex situ populations used as reintroduction sources leads directly to the success or failure of reintroduction, and individuals used for reintroduction should have high genetic diversity (Frankham et al. 2010). Optimization analysis revealed that additional seed sources for ex situ populations of Polemonium kiushianum have to be collected from all five wild populations to preserve all alleles. Moreover, the simulation of seed transfer from wild to ex situ populations demonstrated that more than 1,000 seeds were needed to achieve ex situ population genetic composition that closely approximated that of the wild populations. These results indicated that the transfer of many seeds from wild to ex situ

376 populations could be useful for enhancing genetic diversity in ex situ populations.

378 population demographics by seed removal. Demographic models of the effects of seed

379 collection on extinction risk of 22 perennial species revealed that harvesting $10 \%$ of seeds

380 typically does not increase their extinction risks (Menges et al., 2004). The estimated mean

381 value and standard deviation of the number of seeds per individual of P. kiushianum was $412 \pm$

382430 (Yokogawa et al., unpublished data). Collecting less than 40 seeds from each wild

383 individual would result in less than $10 \%$ of seeds being collected, which may have little impact

384 on population viability. Additionally, the transfer of a small number of seeds from wild to ex situ 
385 populations every year would be a useful approach for enhancing the genetic diversity of ex situ

386 populations, while at the same time minimally impacting the viability of wild populations. This

387 constant immigration from wild to ex situ population would also reduce the rate of genetic

388 adaptation to the ex situ environment (Woodworth et al., 2002).

389 In general, using local seed sources to maximize local adaptation and prevent out

390 breeding depression is recommended in the restoration of endangered species (Mijnsbrugge et

391 al., 2010, Aavik et al. 2012). However, strict use of local seed sources can decrease the

392 availability of high-quality seeds for restoration in highly modified landscapes (Broadhurst et al.,

393 2008). We conducted seed transfer simulation with random seed collection from all wild

394 populations; in other words, each population was not treated separately because the genetic

395 differentiation observed in P. kiushianum is likely to be caused by habitat fragmentation with

396 highly fragmented grassland landscapes. However, contributing factors to genetic differentiation

397 vary among different endangered species. The management and conservation strategy of genetic

398 diversity in ex situ populations have to be determined based on the genetic data of the target

399 species and its surrounding landscape.

400 Given the extinction crisis that is occurring throughout the world, ensuring the

401 maintenance of genetically viable ex situ populations of endangered species is crucial. This

402 study shows that the simulation method using simulated genotypes can be used to aid

403 conservation programs for critically endangered species based on genotype data. Most

404 importantly, the effectiveness of any transfer of individuals or seeds can be simulated before the

405 removal of seeds from wild populations is undertaken. These conservation approaches could

406 provide a means to ensure efficient genetic management of ex situ populations.

407

408 5. Conclusions 
The findings of the present study have important implications for the conservation

management of Polemonium kiushianum and other critically endangered species both in situ and

411 ex situ. Our results show that despite the differences in population sizes, the levels of genetic

412 diversity in all the remaining wild populations were similar to each other. Moreover, these

413 populations underwent genetic differentiation and severe drift associated with habitat

414 degradation, and show decreased gene flow between the remnant populations. The removal of

415 barriers to gene flow and improvement of connectivity between remnant populations will be the

416 priority for the conservation of this species. In other words, it is important to restore grasslands

417 including the habitat of this species, by restarting the management of grasslands and removing

418 artificial conifer plantations that have fragmented grasslands in the study area.

419 While the ex situ populations of P. kiushianum have a lower genetic diversity and

420 different genetic composition compared with those of the wild populations, seed transfer

421 simulations revealed that seed transfer from wild populations would be a useful approach to

422 enhance the genetic diversity of ex situ populations. These results also indicated that more than

4231,000 seeds were needed to achieve the desired genetic composition in ex situ populations. We

424 recommend the constant immigration of seeds from all wild populations to ex situ populations

425 to maintain genetic diversity and ability of ex situ populations to serve as reintroduction sources.

426 Seed source collections without genetic information cause loss of genetic diversity and/or

427 changes in genetic composition in ex situ conservation (Li et al. 2005; Enßlin et al. 2011).

428 Before seed transfer management or foundation of ex situ populations, it is preferable to predict

429 their effects to preserve the genetic diversity of endangered species in ex situ conservation.

\section{Appendix A. Supplementary material}


433 A.1) and pairwise $F_{\text {ST }}$ values and geographic distance among populations (Table A. 2).

434

435

\section{Acknowledgments}

436

We thank T. Abe, N. Fujii, M. Kunimura, S. Nagahara, S. Sei, M. Takehara, T.

Tsurubayashi, K. Uno and N. Uno for their helpful support of sampling. We also thank J. Worth,

M. Yamasaki and two anonymous reviewers for their valuable discussions and helpful supports on this manuscript. This work was supported by Grants in Aid for Scientific Research (20241056) and Grant in Aid for JSPS Fellows (10J03350) from the Japan Society for the Promotion of Science (JSPS).

Reference

Aavik T., Edwards, P.J., Holderegger, R., Graf, R., Billeter, R., 2012. Genetic consequences of using seed mixtures in restoration: A case study of a wetland plant Lychnis flos-cuculi. Biological Conservation 145, 195-204.

Ball, I.R., H.P. Possingham, and M. Watts. 2009. Marxan and relatives: Software for spatial conservation prioritisation. In: Moilanen, A., Wilson, K.A., Possingham, H.P., (eds) Spatial conservation prioritisation: Quantitative methods and computational tools. Oxford University Press, Oxford, pp. 185-195.

Broadhurst, L.M, Lowe, A., Coates, D.J., Cunningham, S.A., McDonald, M., Vesk, P.A., Yates, C., 2008. Seed supply for broadscale restoration: maximizing evolutionary potential. Evolutionary Applications 1, 587-597. recent population bottlenecks from allele frequency data. Genetics 144, 2001-2014. 
457

458

459

460

461

462

463

464

465

466

467

468

469

470

471

472

473

474

475

476

477

478

479

480

Lima, J.de.S., Dobrovolski, R., Chaves, L.J., Naves, R.V., Loyola, R.D., Telles, M.P.C., 2012. Planning for optimal conservation of geographical genetic variability within species. Conservation Genetics 13, 1085-1093.

El Mousadik, A., Petit, R.J., 1996. High level of genetic differentiation for allelic richness among populations of the argan tree [Argania spinosa (L.) Skeels] endemic to Morocco. Theoretical and Applied Genetics 92, 832-839.

Enßlin, A., Sandner, T.M., Matthies, D., 2011. Consequences of ex situ cultivation of plants: Genetic diversity, fitness and adaptation of the monocarpic Cynoglossum officinale L. in botanic gardens. Biological Conservation 144, 272-278.

Environment Agency of Japan, 2000. Threatened wildlife of Japan ---- Red Data Book - , Vascular plants, second ed. Japan Wildlife Research Center, Tokyo (in Japanese).

Evanno, G., Regnaut, S., Goudet, J., 2005. Detecting the number of clusters of individuals using the software STRUCTURE: a simulation study. Molecular Ecology 14, 2611-2620.

Falush, D., Stephens, M., Pritchard, J.K., 2003. Inference of population structure using multilocus genotype data: linked loci and correlated allele frequencies. Genetics 164, $1567-1678$.

Frankel, O.H., Soulé. M.E., 1981. Conservation and Evolustion. Cambridge University Press, Cambridge.

Frankham, R., Ballou, J.D., Briscoe, D.A., 2010. Introduction to conservation genetics, second ed. Cambridge Universtiy Press, Cambridge.

Goudet, J., 2001. FSTAT; a program to estimate and test gene diversities and fixation indices version 2.9.3. <http://www2.unil.ch/popgen/softwares/fstat.htm> (accessed 12.01.12).

Hedrick, P.W., 2005. A standardized genetic differentiation measure. Evolution 59, 1633-1638.

Honnay, O., Adriaens, D., Coart, E., Jacquemyn, H., Roldan-Ruiz, I., 2007. Genetic diversity 
within and between remnant populations of the endangered calcareous grassland plant

Hotta, M. 1974. Evolutionary Biology in Plants: History and Geography of Plants. Sanseido, Tokyo (in Japanese).

Hollingsworth, P.M., Dawson, I.K., Goodall-Copestake, P., Richardson, J.E., Weber, J.C., Sotelo Montes, C., Pennington, T., 2005. Do farmers reduce genetic diversity when they domesticate tropical trees? A case study from Amazonia. Molecular Ecology 14, 497-501.

Hubisz, M.J., Falush, D., Stephens, M., Pritchard, J.K., 2009. Inferring weak population structure with the assistance of sample group information. Molecular Ecology Resources 9, 1322-1332.

Husband, B.C., Campbell, L.G., 2004. Population responses to novel environmental: implications for ex situ plant conservation. In: Guerrant, E.O., Havens, K., Maunder, M. (Eds.), Ex Situ Plant Conservation: Supporting Species Survival in the Wild. Island Press, Washington, pp. 231-266.

Inaba, K., Matoba, H., Nagano, K., Uchiyama, H., 2010. Cytological studies of the critically endangered plants in Japan (1) Polemonium kiushianum (Polemoniaceae). Journal of Japanese Botany 85,118-121.

499 Jacquemyn, H., Roldan-Ruiz, I., Honnay, O., 2010. Evidence for demographic bottlenecks and limited gene flow leading to low genetic diversity in a rare thistle. Conservation Genetics 11, 1979-1987.

Keller, L.F., Waller, D.M., 2002. Inbreeding effects in wild populations. Trends in Ecology

504 Knight, M.E., Martin, A.P., Bishop, S., Osborne, J.L., Hale, R.J., Sanderson, A., Goulson, D., 
2005. An interspecific comparison of foraging range and nest density of four bumblebee (Bombus) species. Molecular Ecology 14, 1811-1820.

Leimu, R., Mutikainen, P., Koricheva, J., Fischer, M., 2006. How general are positive relationships between plant population size, fitness and genetic variation? Journal of Ecology 94, 942-952.

Li, Q., Zu, Z., He, T., 2002. Ex situ genetic conservation of endangered Vatica guangxiensis (Dipterocarpaceae) in China. Biological conservation 106, 151-156. between wild and artificial populations of Metasequoia glyptostroboides: Implications for species recovery. Conservation Biology 19, 224-231.

Liu, M.H., Chen, X.Y., Zhang, X., Shen, D.W., 2008. A population genetic evaluation of ecological restoration with the case study on Cyclobalanopsis myrsinaefolia (Fagaceae). Plant Ecology 197, 31-41.

Lowe, A.J., Boshier, D., Ward, M., Bacles, C.F.E., Navarro, C., 2005. Genetic resource impacts of habitat loss and degradation; reconciling empirical evidence and predicted theory for neotropical trees. Heredity 95, 255-273.

Matoba, H., Inaba, K., Nagano, K., Uchiyama, H., 2011. Use of RAPD analysis to assess the threat of interspecific hybridization to the critically endangered Polemonium kiushianum in Japan. Journal of Plant Research 124, 125-130.

Menges E.S., Guerrant, E.O., Hamze S., 2004. Effects of seed collection on the extinction risk of perennial plants. In: Guerrant, E.O., Havens, K., Maunder, M. (Eds.), Ex Situ Plant Conservation: Supporting Species Survival in the Wild. Island Press, Washington, pp. 305-324. 
seed for ecological restoration. Basic and Applied Ecology 11, 300-311.

530 Milligan, B., 1992. Plant DNA isolation. In: Hoelzel A. R. (Eds.). Molecular Genetic Analysis of Populations: A Practical Approach. IRL Press, Oxford, pp. 59-88

Miyabuchi, Y., Sugiyama, S., Nagaoka, Y., 2012. Vegetation and fire history during the last 30,000 years based on phytolith and macroscopic charcoal records in the eastern of Aso Volcano, Japan. Quaternary international 254, 28-35.

Nei, M., 1987. Molecular evolutionary genetics. Columbia University Press, New York.

Nielsen, E.E., Bach, L.A., Kotlichi, P., 2006. HYBRIDLAB (version 1.0): a program for generating simulated hybrids from population samples. Molecular Ecology Notes 6, 971-973.

Otaki, N, 2000. Germination of buried seeds of Polemonium kiushianum. Botany 50, 82-84. (in Japanese)

Piry, S., Luikart, G., Cornuet, J.M., 1999. BOTTLENECK: A computer program for detecting recent reductions in the effective population size using allele frequency data. Journal of Heredity 90, 502-503.

Possingham, H., Ball, I., Andelman, S., 2000. Mathematical methods for identifying representative reserve networks. In: Ferson, S., Burgman, M., (eds) Quantitative methods for conservation biology . Springer, New York, pp. 291-305.

547 Pritchard, J.K., Stephens, M., Donnelly, P., 2000. Inference of population structure using multilocus genotype data. Genetics 155, 945-959.

Russello, M.A., Amato, G., 2007. On the horns of a dilemma: molecular approaches refine ex situ conservation in crisis. Molecular Ecology 16, 2405-2406. 
Shoji, A., 2006. Conservation of vast grasslands in Aso, Kyushu region. Ecosophia 18, 22-27. (in Japanese)

Takahashi, Y., 2009. Management and restoration of grassland landscape for species conservation: a case of Aso Grassland. The Japanese Institute of Landscape Architecture 72, 394-398. (in Japanese with English summary).

Weir, B.S., Cockerham, C.C., 1984. Estimating f-statistics for the analysis of population structure. Evolution 38, 1358-1370.

Williams, L.W., Serfass, T.L., Cogan, R., Rhodes, O.E., 2002. Microsatellite variation in the reintroduced Pennsylvania elk herd. Molecular Ecology 11, 1299-1310.

Williams, S.L., Davis, C.A., 1996. Population genetic analyses of transplanted eelgrass (Zostera marina) beds reveal reduced genetic diversity in southern California. Restoration Ecology 4, 163-180.

Woodworth, L,M., Montgomery, M.,E., Briscoe, D.,A. Frankham, R., 2002. Rapid genetic deterioration in captive populations: Causes and conservation implications. Conservation Genetics 3, 277-288.

Yokogawa, M., Kaneko, S., Isagi, Y., 2009. Development of microsatellite markers for Polemonium kiushianum (Polemoniaceae), a critically endangered grassland plant species in Japan. Conservation Genetics 10, 1445-1447.

Young, A., Boyle, T., Brown, T., 1996. The population genetic consequences of habitat fragmentation for plants. Trends in Ecology Evolution 11, 413-418. distance. Biological Conservation 143, 669-676. 
577 Table 1. Population characteristics of Polemonium kiushianum examined using microsatellite 578 markers.

579

580

581

582 
583 Table 2. Genetic diversity measurements of each sampled population of Polemonium

584 kiushianum. $N$, numbers of samples; $A$, numbers of alleles per locus; $A R$, allelic richness; $R A$,

585 summed number of rare alleles; $P r$, summed number of private alleles; $H_{\mathrm{O}}$; expected

586 heterozygosity; $H_{\mathrm{E}}$, observed heterozygosity; $F_{\mathrm{IS}}$, fixation index; IAM, infinate allele model;

587 TPM two phase model; N.A., population that was not analysed.

588 
589 Fig 1. (a) Location of the study site, the Aso region. (b) Relative location of the wild

590 populations analyzed in this study. To prevent illegal digging, the precise latitude and longitude,

591 cardinal direction, and topography are not shown in (b).

592

593

594 
595 Fig 2. Results of Bayesian clustering in STRUCTURE analysis (Prichard et al., 2000). (a) Value

596 of $\ln P(X / K)$ for $K=1$ through $K=10 \pm$ SE averaged across 20 runs from the simulation in the

597 STRUCTURE (Prichard et al., 2000). (b) $\Delta K$ based on the rate of change in the log probability

598 of data between successive $K$ values (Evanno et al., 2005). (c) The proportion of the

599 membership coefficient of 182 individuals in seven populations for each of the inferred clusters

600 for $K=3$ and $K=4$. Each column represents an individual.

601

602 
603 Fig 3. Relationship between the number of seeds transferred from wild to ex situ populations

604 and allelic richness (a), expected heterozygosity (b), and inferred cluster of wild populations

605 defined using STRUCTURE analysis (c) for assumed seed transfer populations. The dashed line

606 indicates the value for the total wild population. Genotype data of ex situ population E6 was

607 used.

608

609

610 
611 Table A.1. Characteristics of three new microsatellite loci for Polemonium kiushianum and their

612 variability. Deviation from Hardy-Weinberg equilibrium (HWE) and linkage disequilibrium

613 between loci were tested with FSTAT ver 2.9.3 software (Goudet 2001) using genotype data of

614 population W2. Significance levels were adjusted using Bonferroni correction for multiple

615 testing. Although significant deviations $(P<0.05)$ from HWE were detected for Pkiu965, no

616 significant deviations from HWE were detected for other two loci. There was no evidence of

617 significant linkage disequilibrium between any two of the loci.

618 
619 Table A. 2. Pairwise $F_{\text {ST }}$ values (above diagonal) and pairwise geographic distance (below

620 diagonal) between Polemonium kiushianum populations. The significances were indicated by

621 asterisks $(* P<0.05, * * P<0.01)$.

622 
Table 1.

\begin{tabular}{lccll}
\hline & Wild or & Number of & & \\
Population & flowering & Habitat type & Maintenance \\
& Ex situ & individuals & & \\
\hline W1 & wild & ca. 400 & grasslands & mowing in autumn \\
W2 & wild & ca. 250 & grasslands & mowing in autumn \\
W3 & wild & 3 & edge of conifer plantation & abandonment \\
W4 & wild & 50 & gap of conifer plantation & mowing in autumn \\
W5 & wild & 21 & gap of conifer plantation & abandonment \\
E6 & ex situ & ca. 100 & broad-leaved deciduous forest & mowing in autumn \\
E7 & ex situ & ca. 100 & grassland with deciduous trees & mowing in autumn \\
\hline
\end{tabular}


Table 2.

\begin{tabular}{|c|c|c|c|c|c|c|c|c|c|c|}
\hline \multirow[t]{2}{*}{ Population } & \multirow[t]{2}{*}{$N$} & \multirow[t]{2}{*}{$A$} & \multirow[t]{2}{*}{$A R$} & \multirow[t]{2}{*}{$R A$} & \multirow[t]{2}{*}{$\operatorname{Pr}$} & \multirow[t]{2}{*}{$H_{\mathrm{O}}$} & \multirow{2}{*}{$H_{\mathrm{E}}$} & \multirow[t]{2}{*}{$F_{\mathrm{IS}}$} & \multicolumn{2}{|c|}{$\begin{array}{l}P \text { value of } \\
\text { Bottleneck analysis }\end{array}$} \\
\hline & & & & & & & & & IAM & TPM \\
\hline \multicolumn{11}{|c|}{ Wild populations } \\
\hline W1 & 34 & 3.80 & 2.68 & 4 & 1 & 0.38 & 0.39 & 0.01 & 0.01 & 0.58 \\
\hline W2 & 32 & 3.80 & 2.94 & 4 & 5 & 0.47 & 0.47 & 0.04 & 0.04 & 0.46 \\
\hline W3 & 7 & 2.90 & 2.90 & 2 & 2 & 0.34 & 0.42 & 0.26 & N.A. & N.A. \\
\hline W4 & 32 & 3.70 & 2.71 & 6 & 5 & 0.36 & 0.38 & 0.06 & 0.02 & 0.47 \\
\hline W5 & 21 & 3.20 & 2.56 & 4 & 2 & 0.46 & 0.41 & -0.07 & 0.01 & 0.28 \\
\hline \multicolumn{11}{|c|}{ Ex situ populations } \\
\hline E6 & 32 & 3.10 & 2.45 & 1 & 1 & 0.34 & 0.39 & 0.08 & 0.10 & 0.67 \\
\hline \multirow[t]{2}{*}{ E7 } & 24 & 3.10 & 2.66 & 0 & 0 & 0.35 & 0.37 & 0.06 & 0.15 & 0.71 \\
\hline & average & 3.37 & 2.70 & 3.0 & 2.3 & 0.39 & 0.40 & & & \\
\hline
\end{tabular}


Fig 1.

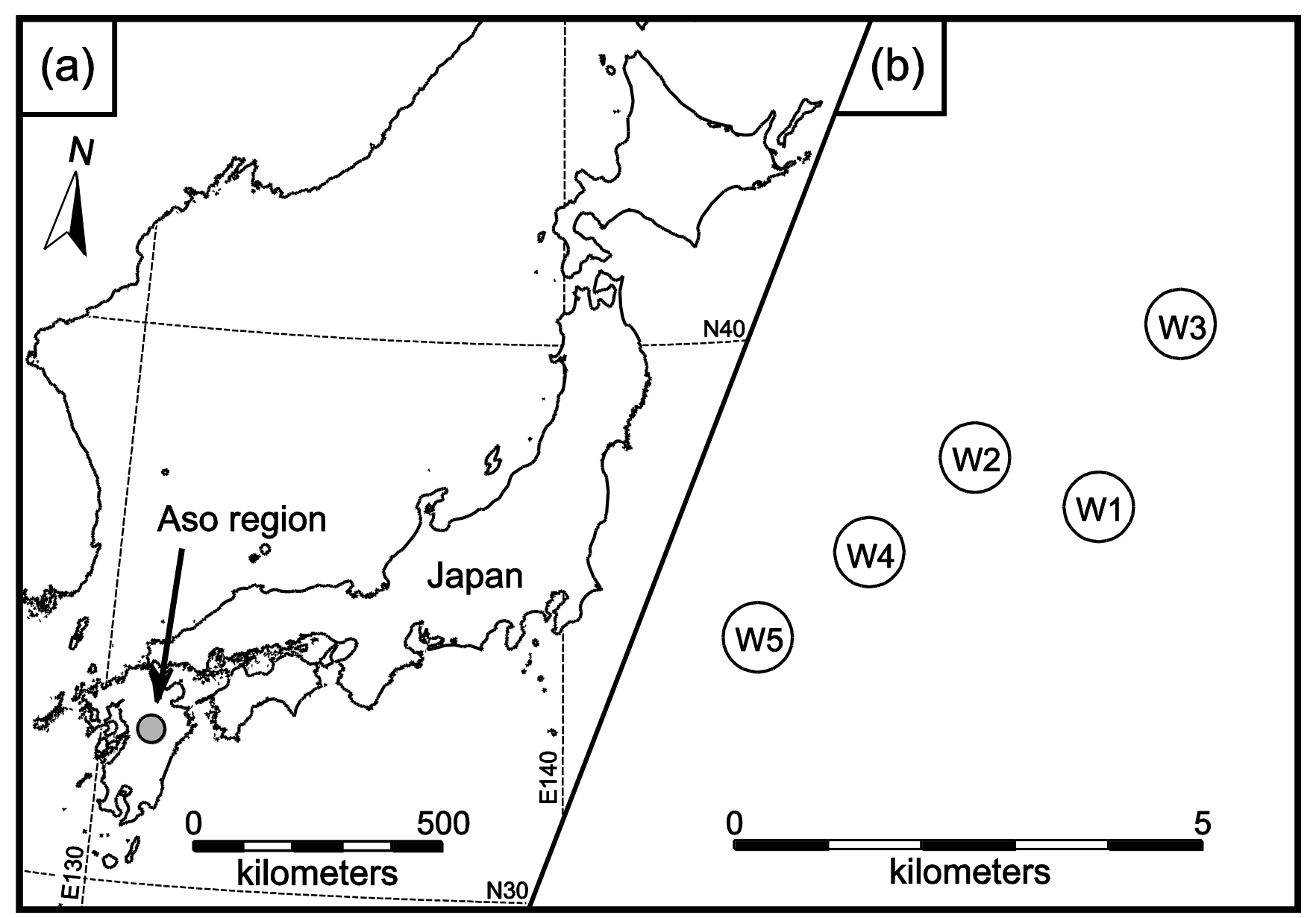


Fig 2.

(a)

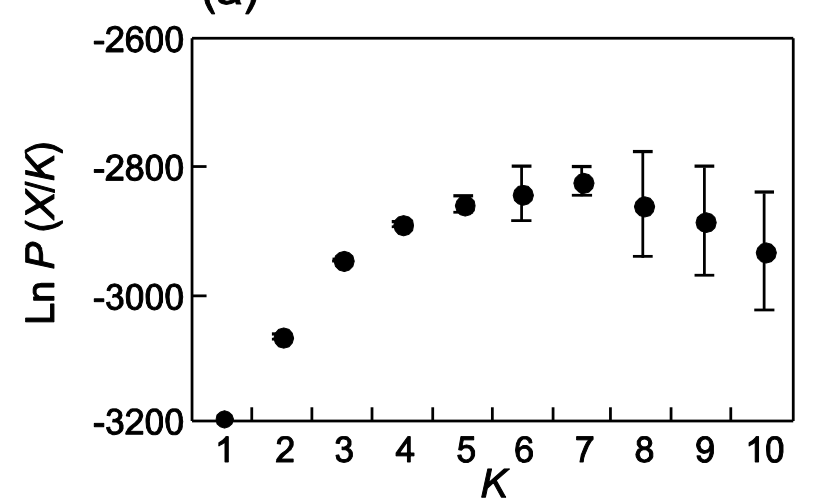

(c)

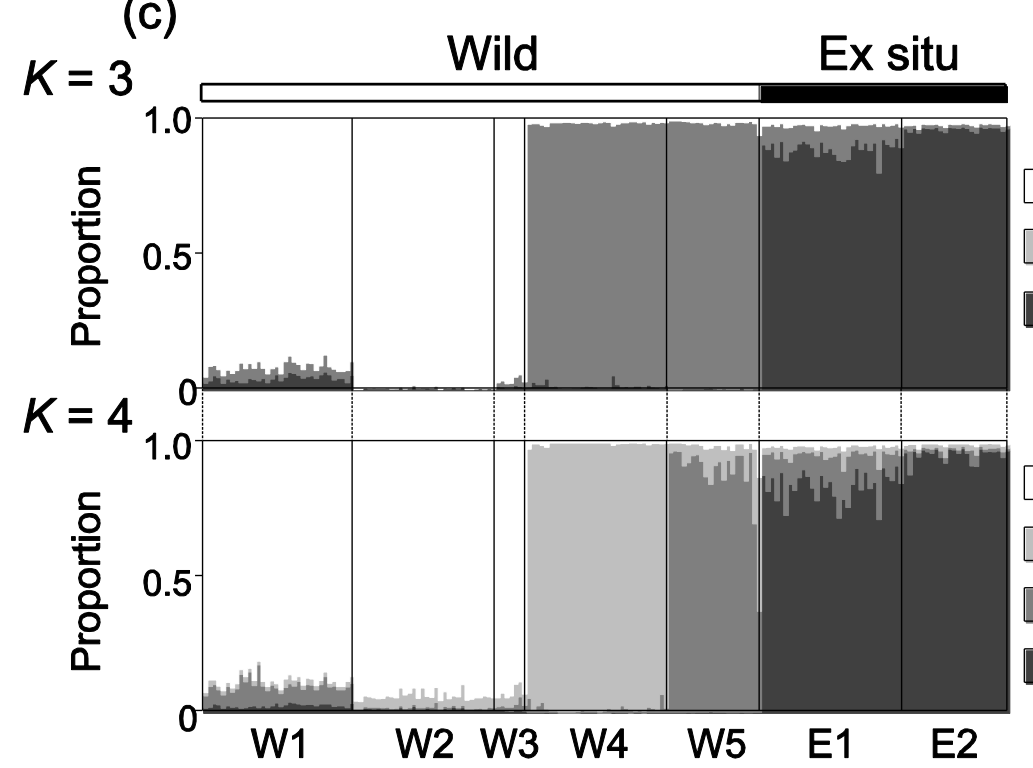

(b)

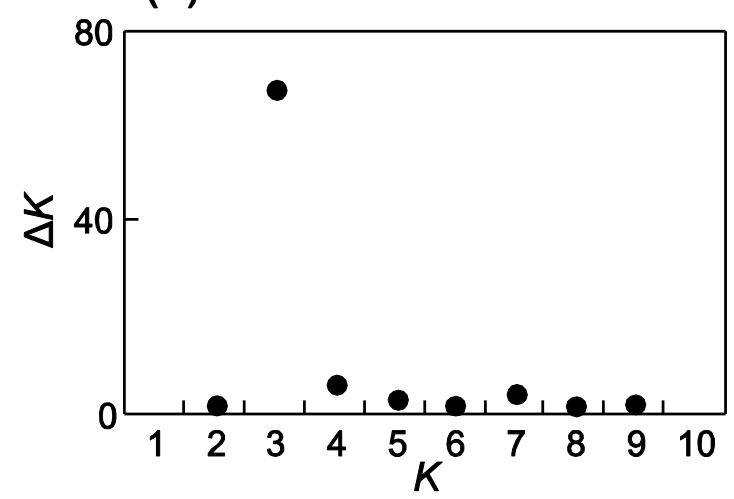

$\square$ Cluster I $F=0.06, H_{\mathrm{E}}=0.46$

$\square$ Cluster II $F=0.10, H_{\mathrm{E}}=0.43$

Cluster III $F=0.21, H_{E}=0.38$

$\square$ Cluster I $F=0.07, H_{\mathrm{E}}=0.45$

$\square$ Cluster II-a $F=0.12, H_{E}=0.39$

$\square$ Cluster II-b $F=0.22, H_{\mathrm{E}}=0.42$

Cluster III $F=0.21, H_{E}=0.38$ 
Fig 3.
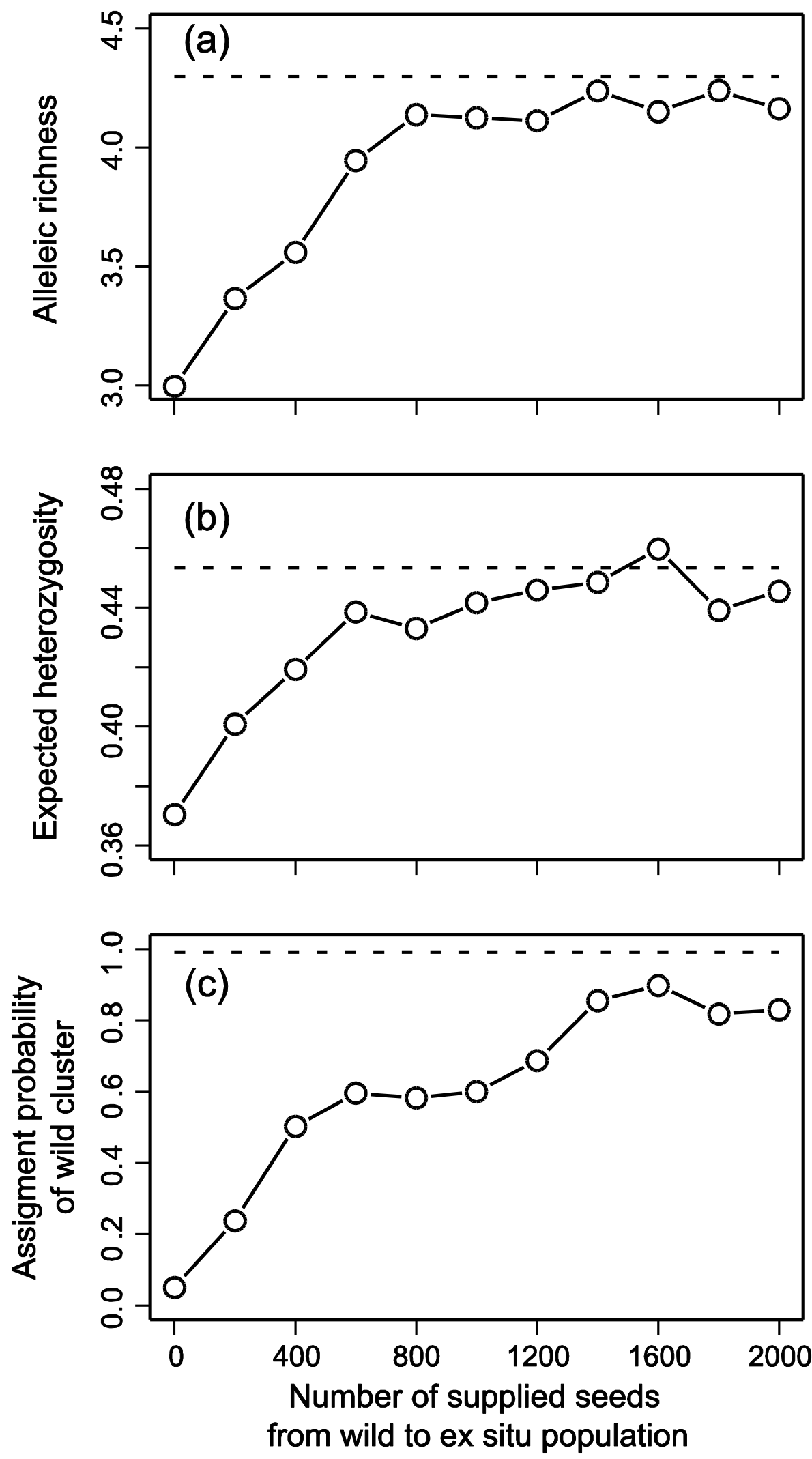
Table A. 1.

\begin{tabular}{|c|c|c|c|c|c|c|c|c|}
\hline Locus & Repeat motif & Primer sequence (5'-3') & $\begin{array}{l}\text { Ta } \\
\left({ }^{\circ} \mathrm{C}\right)\end{array}$ & $\begin{array}{l}\text { Size } \\
\text { range } \\
\text { (bp) }\end{array}$ & $A$ & $H_{\mathrm{O}}$ & $H_{\mathrm{E}}$ & $\begin{array}{l}\text { Accession } \\
\text { number }\end{array}$ \\
\hline \multirow[t]{2}{*}{ Pkiu593 } & $(\mathrm{AG})_{6}(\mathrm{AC})_{11}$ & AGAGAGAGAGAGACACACACAC & 57 & $189-204$ & 4 & 0.688 & 0.604 & AB721308 \\
\hline & & CAGACAACTCCATGTTTGAGAT & & & & & & \\
\hline \multirow[t]{2}{*}{ Pkiu627 } & $(\mathrm{AC})_{6}(\mathrm{TC})_{7}$ & АСАСАСАСАСАСТСТСТСТСТС & 57 & $257-275$ & 3 & 0.129 & 0.177 & AB721309 \\
\hline & & GAGGGACAGAGAGATCAAGAAC & & & & & & \\
\hline \multirow[t]{2}{*}{ Pkiu965 } & $(\mathrm{AG})_{6}(\mathrm{AC})_{10}$ & AGAGAGAGAGAGACACAC & 45 & $154-174$ & 4 & $0.483 *$ & 0.699 & AB721310 \\
\hline & & TAATAGTCATAAAATAAGAGGT & & & & & & \\
\hline
\end{tabular}


Table A. 2. Pairwise $F_{\mathrm{ST}}$ values (above diagonal) and pairwise geographic distance (below diagonal) between Polemonium kiushianum populations.

The significances were indicated by asterisks $(* P<0.05$, ** $P<0.01)$.

\begin{tabular}{lllllllll}
\hline & \multicolumn{3}{c}{ Wild } & & & & & \multicolumn{2}{c}{ Restored } \\
\cline { 2 - 3 } \cline { 8 - 9 } Population & W1 & W2 & W3 & W4 & W5 & & R6 & R7 \\
\hline W1 & --- & $0.03^{*}$ & $0.07^{* *}$ & $0.09^{* *}$ & $0.12^{* *}$ & & $0.10^{* *}$ & $0.10^{* *}$ \\
W2 & 1.4 & --- & $0.08^{* *}$ & $0.08^{* *}$ & $0.11^{* *}$ & & $0.10^{* *}$ & $0.09^{* *}$ \\
W3 & 2.1 & 2.7 & --- & $0.17^{* *}$ & $0.18^{* *}$ & & $0.21^{* *}$ & $0.18^{* *}$ \\
W4 & 2.5 & 1.5 & 4.2 & -- & $0.13^{* *}$ & $0.09^{* *}$ & $0.08^{* *}$ \\
W5 & 4.0 & 3.0 & 5.7 & 1.51 & -- & & $0.14^{* *}$ & $0.16^{* *}$ \\
R6 & 6.9 & 6.4 & 5.3 & 7.45 & 8.49 & & --- & $0.08^{* *}$ \\
R7 & 32.2 & 33.6 & 31.8 & 34.5 & 35.6 & & 35.5 & --- \\
\hline
\end{tabular}

